\title{
DESIGN AND FEEDBACK CONTROL OF ELECTROSTATIC ACTUATORS FOR MAGNETIC DISK DRIVES
}

\author{
David A. Horsley ${ }^{*}$, Naiyavudhi Wongkomet, Roberto Horowitz, and Albert P. Pisano \\ Berkeley Sensor and Actuator Center \\ 497 Cory Hall, University of California at Berkeley, CA 94720
}

\begin{abstract}
This paper presents a microfabricated actuator designed for high precision servo-positioning in a magnetic hard disk drive. The device is actuated using electrostatic force generated with parallelplate capacitive electrodes. The displacement of these electrodes is measured using a dedicated capacitive sensing interface, allowing closed-loop control to be used to extend the servo bandwidth. Using the sensing electronics and a simple phase-lead compensator, a prototype device was used to actuate a $1.6 \mathrm{mg}$ ceramic slider over a $1.2 \mathrm{kHz}$ bandwidth.
\end{abstract}

\section{INTRODUCTION}

The magnetic hard disk drive continues to maintain a significantly lower cost per megabit in comparison to competing solidstate memory technology. One means of maintaining this advantage is by increasing the data storage density by reducing the size of each data bit, requiring increased accuracy in positioning the $\mathrm{read} / \mathrm{write}$ heads over the disk. To this end, a dual-stage positioning mechanism has been proposed, using a conventional voice-coil motor (VCM) for coarse positioning and a high bandwidth secondary actuator for fine positioning [1]. In this paper we will describe a microfabricated secondary actuator which is only slightly larger than the ceramic block, referred to as the slider, which bears the magnetic read/write heads in a disk drive. This actuator is designed to carry the slider and will itself be mounted on the VCM-actuated arm upon which the slider is usually attached. The advantage to this approach over competing designs is that the actuators are batch-fabricated for low cost, but are not expected to significantly effect either the head or suspension fabrication processes. Electrostatic actuation has been selected for ease of fabrication, since the structural material need only be conductive, rather than ferromagnetic or piezoelectric. Previously, we described a rotational, microfabricated actuator for this purpose [2].

\section{ACTUATOR DESIGN}

The actuator, illustrated in Fig. 1, consists of a translating central shuttle which is anchored to fixed stator segments via a flexural suspension. Actuation is achieved by electrostatic force generated using capacitive electrodes which are mounted in opposing pairs between the shuttle and stators. The device is quadsymmetric, meaning that the upper stator segments are used to pull the shuttle in the $+x$ direction, while the lower segments are used to push the shuttle in the - $x$ direction. Because the disk-drive application requires high output force over a relatively small displacement range $( \pm 1 \mu \mathrm{m})$, parallel plate capacitors have been selected rather than interdigitated ("comb-finger") electrodes. The main drawback to this configuration is that the electrostatic attraction between parallel-plate capacitors is a nonlinear function of the separation between the plates. However, assuming a nominal capacitive gap of $g_{0}$ and an electrode length of $l_{p}$, the ratio of the parallel-plate force to the interdigitated force is $l_{p} / g_{0}$. Since typical electrode lengths are over ten times greater than the capacitive gap, the parallel plate configuration enjoys an order of magnitude greater output force than an interdigitated design with the same area and capacitive gap. It is well known that a voltage driven parallel plate has a stable actuation range of $\pm g_{0} / 3$; at larger displacements the electrostatic force dominates over the restoring force from the suspension and the electrodes pull together. To ensure that the actuator will not exceed this range under normal operation, a nominal capacitive gap of $10 \mu \mathrm{m}$ was selected, allowing a $\pm 3.3 \mu \mathrm{m}$ stable actuation range. Furthermore, to prevent the electrodes from coming into contact due to a large external shock, fixed stoppers which are biased at the same potential as the shuttle are placed above and below the shuttle, limiting the displacement to a maximum range of $\pm 4 \mu \mathrm{m}$. Due to the fact that the actuator is composed of conductive, doped polysilicon, it is currently possible to implement only minimal electrical isolation between components, so each shuttle electrode is bordered by two stator electrodes, both biased at the same potential as illustrated in the top of Fig. 1. The effect of the parasitic capacitive gap, $\alpha g_{0}$, is to diminish the electrostatic force generated from the primary capacitive gap, $g_{0}$. Increasing $\alpha$ results in a greater net force on each shuttle capacitor but reduces the total number of capacitors per unit length. As proposed in [3], an optimization was performed to maximize the total actuation force, resulting in a value of $\alpha=2.2$.

\section{FABRICATION PROCESS}

Actuators were fabricated using a modified version of the HexSil process first described in [4]. The advantage of this process is that it produces high aspect-ratio structures with heights of up to $100-200 \mu \mathrm{m}$ using a molded, $4 \mu \mathrm{m}$ thick, CVD polysilicon film. Molds are fabricated by etching deep trenches into standard (100) silicon wafers using deep reactive ion etching (DRIE). Since the depth of these trenches determines the height of the finished actuators, the mold DRIE is a critical process. Prototype molds were limited to $50 \mu \mathrm{m}$ depths, but future devices will have doubled thickness and output force. Following the mold fabrication, the mold is filled with in-situ phosphorous doped CVD polysilicon on top of a $\mathrm{CVD} \mathrm{SiO}_{2}$ sacrificial layer. To ensure conformality of the $\mathrm{SiO} 2$ film it is formed by thermally oxidizing a conformal CVD polysilicon layer. The surface polysilicon is then patterned and metallized using conventional surface micromachining techniques. A cross-sectional optical micrograph of the filled trenches is shown in Fig. 2. Note that there are bright bands visible in the sacrificial layer due to incomplete oxidation of the polysilicon. The completed structures are released from the mold wafer by an etch in hydrofluoric acid (HF), which removes the sacrificial layer. The actuators are then assembled onto copper interconnect on a target

* Present address: DiCon Fiberoptics, 1331 Eighth St., Berkeley, CA 94710 


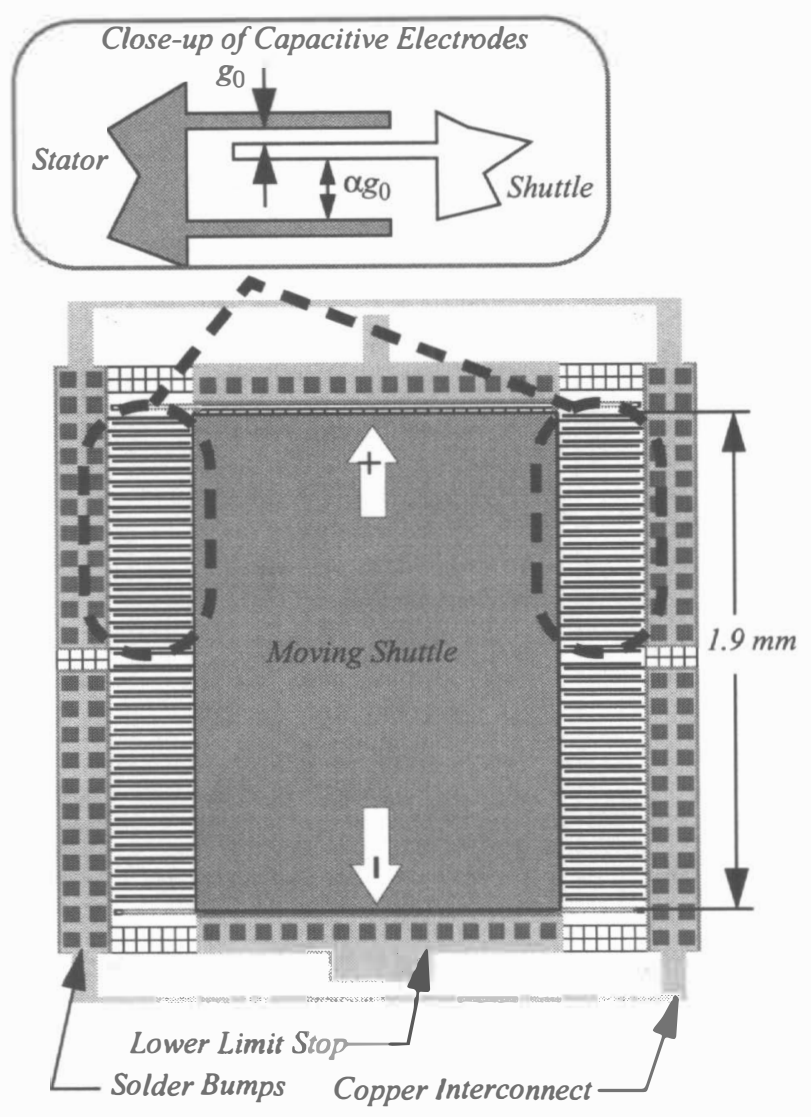

Figure 1. Actuator layout. The upper stator segments (circled) serve to pull the shuttle in the $+x$ direction, while the symmetric lower stator electrodes push the shuttle in the $-x$ direction.

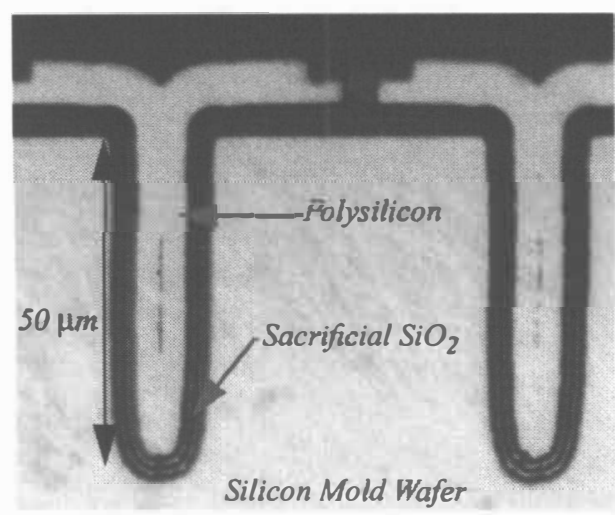

Figure 2. Optical micrograph of filled mold trenches. Note that slight bowing of the mold trenches has resulted in voids in the polysilicon beams. Also, bright bands in the sacrificial layer are observed due to incomplete oxidation.

substrate using a solder bump bonding process described in [5]. Due to the fact that the structural material is uniformly conductive, electrical isolation is achieved through the use of break-away or fusible beams as illustrated in Fig. 3 .

\section{ACTUATION DYNAMICS}

For small deflections, $x(t)$, the mechanical behavior of the actuator in response to an applied electrostatic force, $F\left(x, v_{1}\right)$, may be described by a mass-spring-damper model,

$$
m \ddot{x}(t)+b \dot{x}(t)+k_{m} x(t)=F\left(x, v_{1}\right)
$$
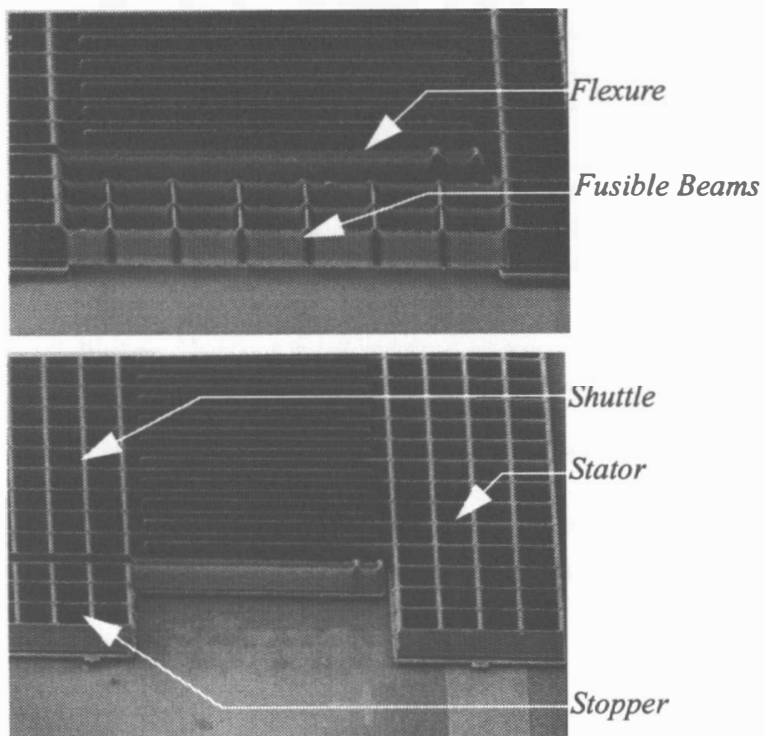

Figure 3. Electrical isolation of assembled actuators. Top: fusible beams connecting the stopper and stator segments. Bottom: the same region after the application of a brief pulse of current.

where $m$ denotes the total mass of the slider and actuator, $b$ denotes the damping coefficient, and $k_{m}$ is the suspension spring constant. Although the electrostatic force, $F\left(x, v_{1}\right)$, is a nonlinear function of both the actuator displacement and the applied voltage, use of pushpull stator segments which are DC biased with a voltage $V_{0}$ and driven with a differential input voltage, $v_{1}$, allows small-signal linearization. A linear approximation for the force is then given by the first-order Taylor-series expansion:

$$
F\left(x, v_{1}\right) \approx k_{e}\left(V_{0}\right) x+k_{v}\left(V_{0}\right) v_{1}
$$

Assuming the capacitor configuration illustrated in Fig. 1, with a nominal capacitive gap of $g_{0}$ and a parasitic capacitive gap of $\alpha g_{0}$ (where $\alpha>1$ ):

$$
\begin{aligned}
& k_{e}\left(V_{0}\right)=\left.\frac{\partial F}{\partial x}\right|_{\left(0, V_{0}\right)}=\frac{2 \varepsilon_{0} A}{g_{0}^{3}}\left(1+\frac{1}{\alpha^{3}}\right) v_{0}^{2} \\
& k_{v}\left(V_{0}\right)=\left.\frac{\partial F}{\partial v_{1}}\right|_{\left(0, V_{0}\right)}=\frac{2 \varepsilon_{0} A}{g_{0}^{2}}\left(1-\frac{1}{\alpha^{2}}\right) V_{0}
\end{aligned}
$$

where $\varepsilon_{0}$ is the permittivity of air and $A$ is the total capacitive area of each stator (neglecting parasitics). Substituting the linearized electrostatic force from Eq. (2) into the actuator equation of motion from Eq. (1) yields a linear actuation model:

$$
m \ddot{x}(t)+b \dot{x}(t)+\left(k_{m}-k_{e}\left(V_{0}\right)\right) x(t)=k_{v}\left(V_{0}\right) v_{1}
$$

Note that $k_{v}$ represents a voltage-to-force gain, while the effect of $k_{e}$ is that of a negative, electrostatic spring. This electrostatic spring term reflects the tendency for the parallel plates to pull together for sufficiently high voltage, which occurs when $k_{e}\left(V_{0}\right) \geq k_{m}$.

For a fixed bias voltage, $V_{0}$, the Laplace transform of Eq. (4) is that of a simple second-order system,

$$
\frac{X(s)}{V_{1}(s)}=\frac{k_{v}\left(V_{0}\right)}{m s^{2}+b s+\left(k_{m}-k_{e}\left(V_{0}\right)\right)}
$$


Typical microfabricated actuators exhibit very lightly damped resonances, implying that if the resonant frequency is near the desired servo bandwidth, the settling performance will be poor. One approach is to design the actuator to have an extremely high resonance frequency so that the actuator dynamics will have negligible effect on the servo system performance. For this approach to succeed, the resonance frequency should be from five to ten times greater than the servo bandwidth. Alternatively, the actuator may be designed to have an open-loop resonant frequency which is well within the control bandwidth. Feedback is then used to provide the desired closed-loop resonant frequency and damping ratio. The advantage of the latter approach is quickly seen by noting that the DC gain of the actuator transfer function listed in Eq. (5) is:

$$
A_{0}=\left(\frac{m}{k_{m}-k_{e}\left(V_{0}\right)}\right)\left(\frac{k_{v}\left(V_{0}\right)}{m}\right)=\frac{1}{\omega_{n}^{2}\left(\frac{k_{v}\left(V_{0}\right)}{m}\right)}
$$

Thus, for a fixed mass and bias voltage, an actuator which has a resonance that is within the control bandwidth will require $100 \times$ lower input voltage to achieve the same displacement as an actuator with a resonant frequency which is ten times higher.

\section{CLOSED-LOOP ACTUATION}

For a second-order system, the closed-loop DC gain and the resonant frequency are related to their open-loop values by:

$$
A_{c l} \omega_{n, c l}^{2}=A_{0} \omega_{n}^{2}=\mathrm{GBW}
$$

where GBW is a constant, analogous to the gain-bandwidth product used to describe first-order systems such as op-amps. Although slight increases in bandwidth may be obtained at the cost of diminished phase-margin, the actuation bandwidth is roughly proportional to the resonant frequency. Thus, feedback can be used to trade DC gain for bandwidth or vice-versa. However, Eq. (7) shows that in order to double the closed-loop bandwidth, the closed-loop DC gain must diminish by a factor of four. The control strategy is to choose the loop gain to achieve the desired closedloop bandwidth, then use phase-lead compensation to yield a desired phase-margin. The block-diagram of the control system is illustrated in Fig. 4.

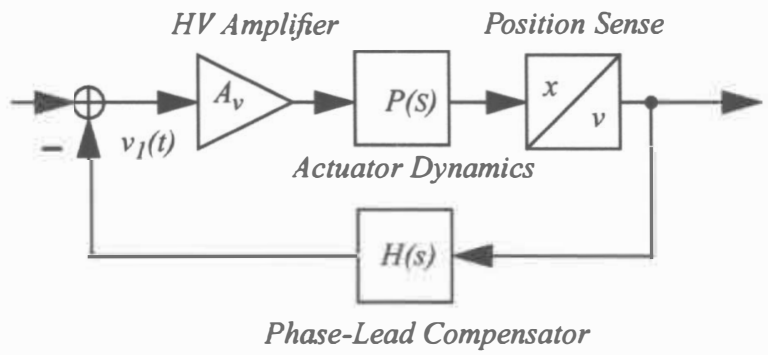

Figure 4. Block-diagram of closed-loop control architecture.

The actuator displacement is measured using a capacitive position sensing interface which is illustrated in Fig. 5. The custom sensing circuit utilizes a pseudo-differential switched-capacitor capacitive sensing scheme [5]. On-chip coupling capacitors shield the sensing electronics from the high-voltage drive signal, which is in the range of $\pm 40 \mathrm{~V}$. Correlated double sampling is used to eliminate amplifier offset, $1 / \mathrm{f}$ noise, switch charge injection, and kT/C noise. To minimize the coupling of the drive signal into the sensing channel, sensing and the driving signals are separated by both frequency division and time division techniques. Unfortunately, this coupling, referred to as feedthough, is not completely suppressed and the minimum detectable signal is limited to approximately $20 \mathrm{~nm}$ using these electronics.

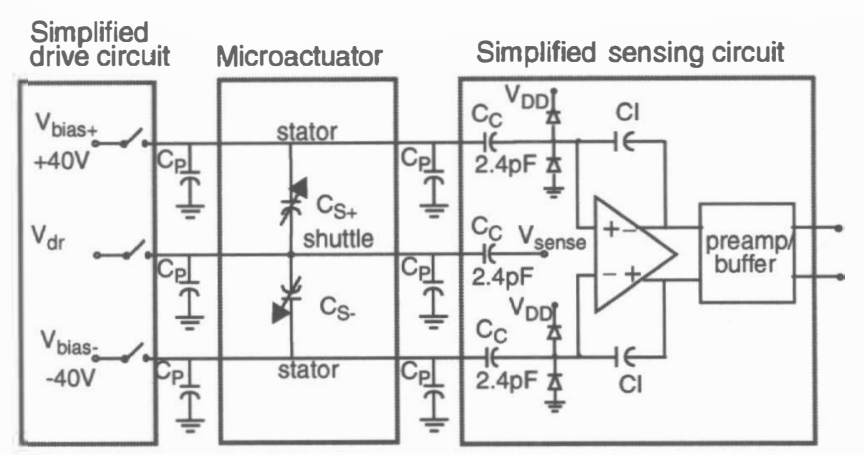

Figure 5. Simplified circuit for capacitive sensing interface.

\section{EXPERIMENTAL RESULTS}

The parameters of the open-loop actuation transfer function listed in Eq. (5) were identified by measuring the actuator frequency response using a laser-doppler vibrometer (LDV). A slider with a measured mass of $1.6 \mathrm{mg}$ was bonded to an actuator, as shown in Fig. 6. Open-loop frequency response measurements taken before and after attaching the slider allowed the moving mass of the actuator to be estimated at $97 \pm 10 \mu \mathrm{g}$. By measuring the resonant frequency and DC-gain at multiple bias voltages, as illustrated in Fig. 7, estimates for the suspension spring constant, $k_{m}$, as well as the electrostatic model parameters, $k_{v}$ and $k_{e}$, were produced. The values estimated from experimental data are listed along with theoretically estimated values in Table 1 . Two different actuator designs were fabricated which were identical except for the fact that one had a larger shuttle than the other. Good agreement is observed between the measured and theoretical values with the exception of the damping coefficient. This error is due to the fact that the theoretical damping model included only viscous gas damping and did not account for added damping due to series resistance between the input amplifier and the capacitive electrodes.

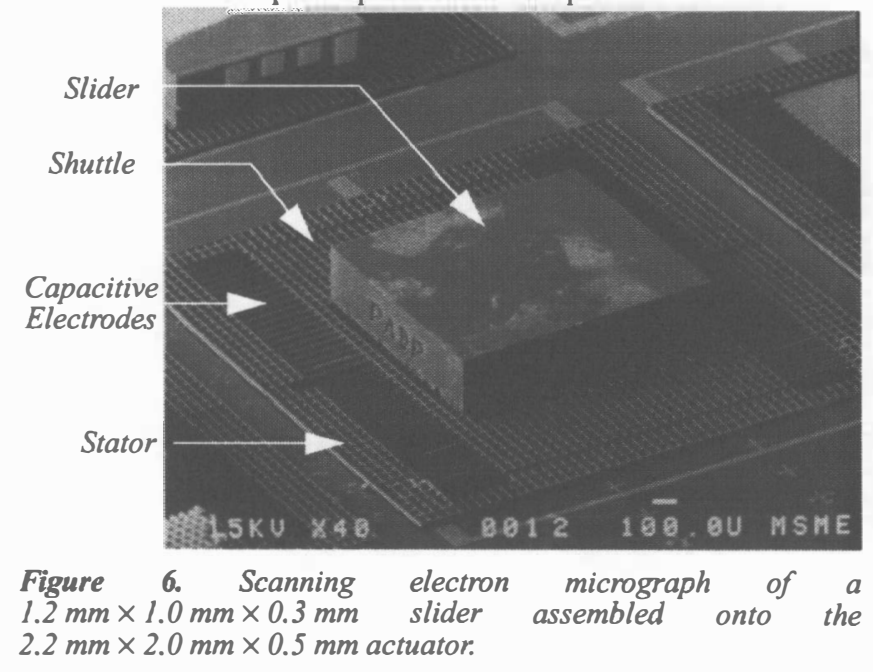

Table 1: Actuation Model Parameters at $V_{0}=40 \mathrm{~V}^{2}$

\begin{tabular}{cccc}
\hline parameter & units & Design \#1 & Design \#2 \\
\hline \multirow{2}{*}{$m$} & $\mu g$ & $97 \pm 10$ & $44 \pm 5$ \\
& \multirow{2}{*}{$\mu \mathrm{(87)}$} & $(46)$ \\
\hline
\end{tabular}



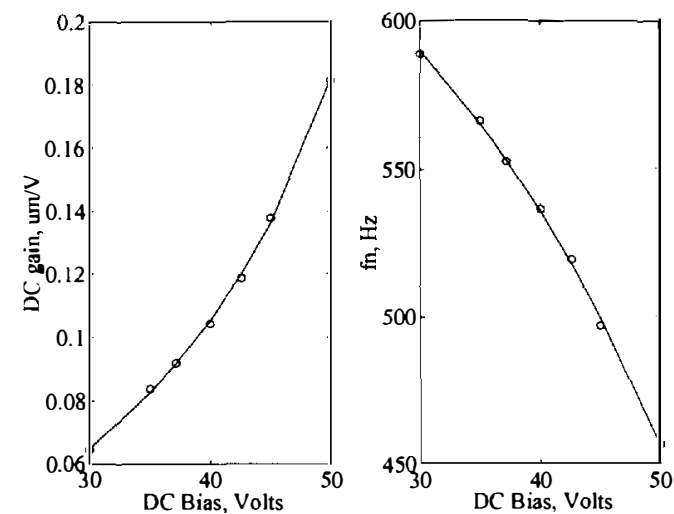

Figure 7. Measured DC gain and resonance freq. vs. bias voltage.

Table 1: Actuation Model Parameters at $V_{0}=40 \mathrm{~V}^{\mathrm{a}}$

\begin{tabular}{cccc}
\hline parameter & units & Design \#l & Design \#2 \\
\hline \multirow{2}{*}{$k_{m}$} & \multirow{2}{*}{$\mathrm{N} / \mathrm{m}$} & $\begin{array}{c}29 \pm 2 \\
(33)\end{array}$ & $\begin{array}{c}29 \pm 2 \\
(33)\end{array}$ \\
\hline \multirow{2}{*}{$b$} & $\mu N-s / m$ & $78 \pm 12$ & $58 \pm 14$ \\
& \multirow{2}{*}{$N / m$} & $9.4 \pm 0.6$ & $9.4 \pm 0.6$ \\
& & $(10.1)$ & $(10.1)$ \\
\hline$k_{e}(40 \mathrm{~V})$ & $\mu N / V$ & $2.0 \pm 0.1$ & $2.0 \pm 0.1$ \\
& & $(2.2)$ & $(2.2)$ \\
\hline$k_{v}(40 \mathrm{~V})$ & &
\end{tabular}

a. Theoretical values appear in parenthesis.

Following the open-loop system identification experiments, closed-loop control was implemented using both LDV measurements and capacitive position sensing. The measured open and closed-loop transfer functions from input voltage to output displacement are shown in Fig. 8, while the closed-loop response to a $100 \mathrm{~Hz}, \pm 10 \mathrm{~V}$ square-wave, measured using capacitive sensing, is plotted in Fig. 9. Note that the higher bandwidth achieved using LDV measurements came at a cost of a four-fold reduction in DC gain, corresponding to a greatly reduced actuation range at $\pm 40 \mathrm{~V}$. A summary of the closed-loop results is provided in Table 2 .

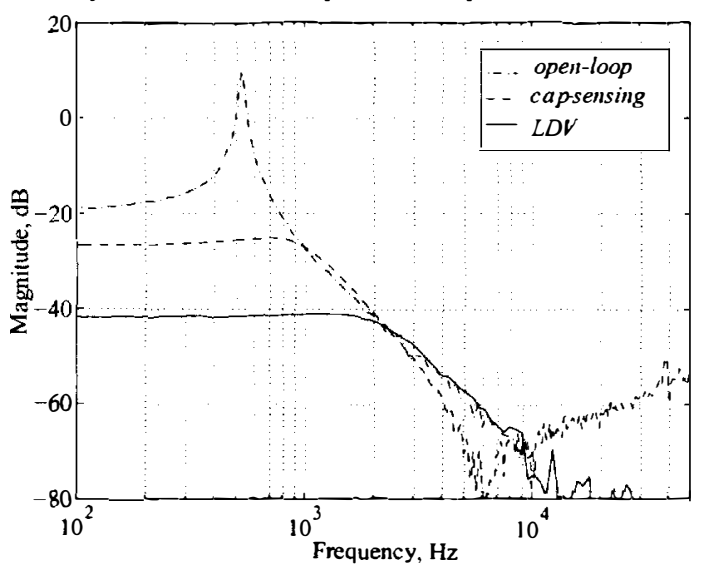

Figure 8. Measured open- and closed-loop frequency response.

\section{CONCLUSIONS}

Closed-loop position control of a disk-drive slider has been demonstrated using a microfabricated actuator. A dedicated capacitive sensing interface was utilized to demonstrate a closed-loop bandwidth of $1.1 \mathrm{kHz}$, although reducing the DC gain by a factor of

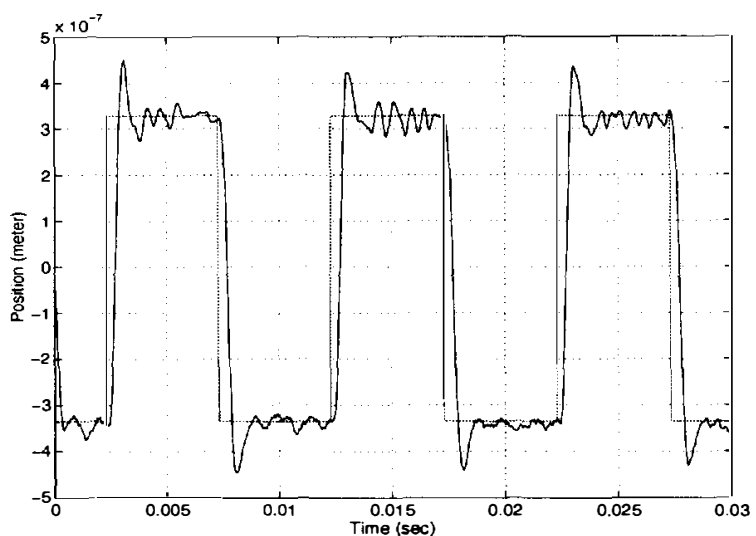

Figure 9. Measured closed-loop response to a $100 \mathrm{~Hz}$ square-wave.

Table 2: Summary of Closed-Loop Results

\begin{tabular}{ccccc}
\hline & $\begin{array}{c}\text { DC gain, } \\
\mu m / V\end{array}$ & $\begin{array}{c}B W, \\
H z\end{array}$ & $\begin{array}{c}\text { Phase } \\
\text { Margin, } \\
\text { Deg. }\end{array}$ & $\begin{array}{c}t_{\text {settle }} \\
\text { msec. }\end{array}$ \\
\hline Open-Loop & 0.1 & 820 & NA & 100 \\
\hline LDV & 0.01 & 2474 & 45 & 0.5 \\
\hline Cap. Sensing & 0.035 & 1170 & 38 & 2.0 \\
\hline
\end{tabular}

four allowed a bandwidth of over $2.4 \mathrm{kHz}$ to be achieved. Future devices which have a doubled thickness will allow a proportionate increase in DC gain and capacitive sensing resolution.

\section{ACKNOWLEDGMENTS}

This research was funded by DARPA contract DABT 63-95C-0028. The authors thank Chris Keller, Angad Singh, and Michael Cohn for their help with developing the actuator fabrication process, along with Stephen Williams of Quantum Corporation for providing the pico-sliders.

\section{REFERENCES}

1. L.-S. Fan, H.H. Ottesen, T.C. Reiley, R.W. Wood, "Magnetic Recording-Head Positioning at Very High Track Densities Using a Microactuator-Based, Two-Stage Servo System", IEEE Trans. Ind. Electronics, v 42, pp. 222-233, June 1995.

2. D.A. Horsley, A. Singh, R. Horowitz, A.P. Pisano, "Angular Micropositioner for Disk Drives," Proc. 10th Int. Conf. on MEMS (MEMS ‘97), Nagoya, Japan, pp. 454-459, 1997.

3. T. Imamura, T. Koshikawa, M. Katayama, "Transverse Mode Electrostatic Microactuator for MEMS-Based HDD Slider," Proc. 9th Int. Conf. on MEMS (MEMS '96), San Diego, CA, pp. 216-221, 1996.

4. C.G. Keller and M. Ferrari, "Milli-Scale Polysilicon Structures," 1994 Solid-State Sensor and Actuator Workshop, Hilton Head, SC, pp.132-137.

5. A. Singh, D.A. Horsley, M.B. Cohn, A.P. Pisano, R.T. Howe, "Batch Transfer of Microstructures using Flip-Chip Solder Bump Bonding," Transducers '97, Chicago, IL, pp. 265-268.

6. N. Wongkomet, Ph.D. Dissertation, Department of Electrical Engineering and Computer Science, University of California at Berkeley, May 1998. 